\title{
A HODOGRAPH APPROACH TO THE ROTATIONAL COMPRESSIBLE FLOW OF AN IDEAL FLUID*
}

\author{
BY \\ GIANFRANCO CHIOCCHIA \\ Politecnico di Torino, Turin, Italy
}

\begin{abstract}
The classical hodograph equation for the study of the irrotational compressible flow of an ideal gas is here extended to the rotational case in which an entropy gradient normal to the streamlines is present. The formulation is in terms of Crocco's stream function and leads to an equation differing from that valid for the irrotational case because of an additional nonlinear $t e \mathrm{~m}$. In spite of this nonlinearity an exact solution can be found, describing a class of rotational vortices. Moreover, perturbative solutions can be found for the cas $:$ in which the entropy gradient and the vorticity are small: one of these is here presented and describes the superposition of a constant vorticity distribution to the transonic region of the Ringleb flow.
\end{abstract}

Introduction. In this work the plane stationary flow of an inviscid compressible fluid with variable entropy is investigated by extending the hodograph procedure classically used for the irrotational (homoentropic) case. The fluid being inviscid, entropy variations along the streamlines are excluded (with the only exception of the discontinuous changes due to shock waves) and the normal variations are described by Crocco's theorem

$$
T \nabla S=\nabla h_{0}+\vec{V} \times(\nabla \times \vec{V}) .
$$

Here $T$ is the (absolute) temperature, $S$ and $h_{0}$ the entropy and the stagnation enthalpy per unit mass, respectively, and $\vec{V}$ the local velocity. Equation (1) shows that the entropy gradient normal to the streamlines is related either to a stagnation enthalpy gradient or to a nonzero vorticity $\omega=|\nabla \times \vec{V}|$ or to the both of them.

In the present work $\nabla h_{0}=0$ is assumed and therefore entropy gradients are only related to nonzero vorticity distributions. This is the common situation occurring behind a curved shock wave, where the nonuniform entropy increase across the shock is responsible for $\nabla S$ and for the vorticity distribution.

This kind of flow is described by the Euler equations, which are also the basis for all current numerical procedures of solution. The alternative method, based on the potential formulation, cannot in fact be used when the flow is rotational.

It is, however, still possible to work in terms of stream function, even if at the price of redefining it in a slightly different way when compared to the classical one

${ }^{*}$ Received November 1, 1988.

(C)1989 Brown University 
[1]. As for the irrotational case, it is then possible to derive a hodograph equation for this new stream function which (in spite of some nonlinear terms now appearing) enables investigations and computations. This equation is deduced in Sec. 1; it turns out to be the extension of the classical Chaplygin equation (see [2], p. 45) for the irrotational case, where now a right-hand side appears. The latter is nonlinear and contains the functional dependence of the entropy on the stream function.

A very peculiar exact solution is given in Sec. 2, representing a class of compressible rotational vortices: they should be compared to the compressible irrotational vortex first studied by Taylor [3] on the physical plane and later by Ringleb [4] on the hodograph one.

In Sec. 3 a linearized form of the hodograph equation is obtained for the case of a sufficiently small entropy gradient. An application is presented in Sec. 4 where a simple irrotational transonic flow, namely the Ringleb flow [4], has been selected as a starting point to produce a perturbated rotational flow pattern. This solution is meaningful on the whole compressible region of the field and is fully analytical, which, to the Author's knowledge, is a very rare occurrence in the literature on compressible rotational flows. This should make the computed case presented here interesting in itself; furthermore, these results do not depend on the peculiar features of some numerical discretization schemes, so that, in spite of their not representing a very practical case, they are nevertheless reliable to be used as benchmarks for the assessment of numerical Euler solvers.

Lastly, it should be remembered that the hodograph method rests on a valid analytical basis: this suggests also that the numerical exploitation of the stream function equation obtained here still deserves consideration.

1. The hodograph equation for a compressible rotational flow. The starting point for this work is the stream function equation on the physical plane $(x, y)$ for a compressible gas flow with variable entropy as given by $\mathrm{L}$. Crocco [1]:

$$
\left(1-\frac{u^{2}}{c^{2}}\right) \Psi_{x x}-2 \frac{u v}{c^{2}} \Psi_{x y}+\left(1-\frac{u^{2}}{c^{2}}\right) \Psi_{y y}=\left(1-w^{2}\right)^{1+2 \beta}\left(\frac{w^{2}}{c^{2}}-1\right) f(\Psi) .
$$

Here all velocities (namely the local speed $w$, its $x, y$-components $u$ and $v$, and the local sound speed $c$ ) are referred to the limiting speed. Therefore, if $\beta$ designates the quantity

$$
\beta=\frac{1}{\gamma-1}
$$

where $\gamma$ is the specific heat ratio, the local sound speed is given by

$$
c^{2}=\frac{1}{2 \beta}\left(1-w^{2}\right) \text {. }
$$

The stream function $\Psi(x, y)$ is the one introduced by Crocco such that

$$
\begin{aligned}
& \Psi_{x}=-v\left(1-w^{2}\right)^{\beta} \\
& \Psi_{y}=u\left(1-w^{2}\right)^{\beta}
\end{aligned}
$$

in order to coincide with the ordinary stream function when the flow is irrotational. In fact it is in this case

$$
\rho=\left(1-w^{2}\right)^{\beta}
$$


everywhere on the flow field, $\rho$ being the local density referred to its stagnation value. If the flow is rotational, Eq. (5) and the analogous one for the pressure $p$

$$
p=\left(1-w^{2}\right)^{\beta+1}
$$

hold only along each streamline because the reference (stagnation) values for $\rho$ and $p$ depend on $\Psi$. Only the relation for the temperature referred to its stagnation value

$$
T=1-w^{2}
$$

still holds everywhere if $h_{0}$ is assumed to be constant (see the Introduction). The validity of Eq. (3) is of course strictly implied by that of Eq. (6).

The function $f(\Psi)$ appearing in Eq. (2) is given by

$$
f(\Psi)=\frac{\omega}{\left(1-w^{2}\right)^{\beta+1}}=\frac{1}{2(\beta+1)} \frac{d}{d \Psi}\left(\frac{S}{R}\right), \quad \omega=|\nabla \times \vec{V}|=v_{x}-u_{y}
$$

being the vorticity, $S$ the (already defined in the Introduction) entropy per unit mass, and $R$ the gas constant from the state equation. It can immediately be seen that if $\omega=0$ or $S=$ constant, then $f(\Psi)=0$ and Eq. (2) reduces to the well-known irrotational form, where also the streamfunction $\Psi$ recovers its classical expression thanks to Eq. (5). An interesting feature of Eq. (2) is that where $w=c$ (sonic condition) the right-hand side vanishes and the flow locally is influencer no more by the entropy gradient.

Lastly, the limit for $w^{2} \ll c^{2}$

$$
\Delta \Psi=-\omega
$$

leads to the classical stream function equation for the rotational flow of an incompressible fluid.

The problem of transferring Eq. (2) onto the hodograph plane to obtain an equation for $\Psi=\Psi(u, v)$ cannot be solved here by the classical method of MoelembroekChaplygin [2] because it requires the existence of a potential function as well. A more universal way would consist in using a Legendre transformation, but this leads to a transformed stream function $\Omega$ whose practical utility is very limited; moreover, Eq. (2) in the present problem explicitly contains a function of $\Psi$, Eq. (7), which makes a formulation in $\Psi$ particularly attractive.

This aim is achieved here through two successive steps: first, an equation in $\Omega$ is obtained; second, this equation is transformed in one in $\Psi$.

Let us define the Legendre-transformed stream function as

$$
\Omega(r, s)=-s x+r y-\Psi(x, y)
$$

where $s$ and $r$ have the following expressions

$$
\begin{gathered}
r=\Psi_{y}=u\left(1-w^{2}\right)^{\beta} \\
s=-\Psi_{x}=v\left(1-w^{2}\right)^{\beta} .
\end{gathered}
$$

It follows directly from Eq. (8)

$$
\begin{aligned}
& \Omega_{r}=y(r, s) \\
& \Omega_{s}=-x(r, s) .
\end{aligned}
$$


The transformation of Eq. (2) can now be performed through a standard procedure (see e.g. [2], p. 36) to obtain

$$
\left(1-\frac{r^{2}}{a^{2}}\right) \Omega_{r r}-2 \frac{r s}{a^{2}} \Omega_{r s}+\left(1-\frac{s^{2}}{a^{2}}\right) \Omega_{s s}=2 \beta J(r, s) a^{2}\left(\frac{r^{2}+s^{2}}{a^{2}}-1\right) f(\Psi)
$$

where $\Psi$ is now given by Eq. ( 8$)$ and $J(r, s)$ is the Jacobian of the transformation

$$
J(r, s)=\frac{\partial(x, y)}{\partial(r, s)}=\Omega_{s s} \Omega_{r r}-\Omega_{r s}^{2}=x_{r} y_{s}-x_{s} y_{r}
$$

(which at this stage can be supposed to be nonzero) and $a$ is a modified sound speed

$$
a=c\left(1-w^{2}\right)^{\beta} \text {. }
$$

In the irrotational case equations are simpler if written in terms of the speed $w$ and its angle $\theta$ to a given direction instead of $u$ and $v$. The reason for this is that the density $\rho$ appearing in the definition of $\Psi$ is a function of $w$ rather than its components. The same simplification is expected to occur here because $\rho$ is substituted through $\left(1-w^{2}\right)^{\beta}$ which is also depending only on $w$. Let us therefore introduce the polar variables

Inversely

$$
\begin{gathered}
q=\left(r^{2}+s^{2}\right)^{1 / 2}=w\left(1-w^{2}\right)^{\beta} \\
\theta=\arctan \frac{s}{r}=\arctan \frac{v}{u} .
\end{gathered}
$$

so that, from Eq. (9)

$$
\begin{aligned}
& r=q \cos \theta \\
& s=q \sin \theta
\end{aligned}
$$

$$
\begin{gathered}
x=-\Omega_{q} \sin \theta-\frac{\omega_{\theta}}{q} \cos \theta \\
y=\Omega_{q} \cos \theta-\frac{\omega_{\theta}}{q} \sin \theta .
\end{gathered}
$$

In the new variables $(q, \theta)$ Eq. (10) becomes

$$
\left(1-\frac{q^{2}}{a^{2}}\right) \Omega_{q q}+\frac{1}{q} \Omega_{q}+\frac{1}{q^{2}} \Omega_{\theta \theta}=2 \beta J(q, \theta) a^{2}\left(\frac{q^{2}}{a^{2}}-1\right) f(\Psi)
$$

where $J(q, \theta)$ is now

$$
J(q, \theta)=\frac{1}{q^{2}}\left[\Omega_{q q}\left(q \Omega_{q}+\Omega_{\theta \theta}\right)-\frac{1}{q^{2}}\left(q \Omega_{q \theta}-\Omega_{\theta}\right)^{2}\right] .
$$

The relation between $\Psi$ and $\Omega$ turns out to be very simple in the new variables. It can be obtained by introducing Eqs. (12) and (13) into Eq. (8)

$$
\Psi(q, \theta)=q \Omega_{\theta}-\Omega
$$

which can readily be integrated to give $\Omega$ as a function of $\Psi$

$$
\Omega(q, \theta)=q \int \frac{\Psi}{q^{2}} d q
$$

where the arbitrary function of $\theta$ which should be added to the integral of Eq. (16) can be neglected without affecting the generality of the result. 
The equation of the streamlines on the plane $(q, \theta)$ can then be obtained by putting $d \Psi=0$ and using Eq. (15). It results in

$$
\frac{d}{d \theta} \Omega_{q}=\frac{\Omega_{\theta}}{q} .
$$

All these relations are very simple thanks to the new hodograph coordinates $(q, \theta)$ introduced here. This fact is particularly evident if Eqs. (14), (15), and (17) are compared to the corresponding ones for the irrotational case as given on the more customary plane $(w, \theta)$

$$
\begin{gathered}
\Omega_{\theta \theta}+\rho w \frac{d}{d w}\left[\frac{w \Omega_{w}}{\rho\left(1-w^{2} / c^{2}\right)}\right]=0 \\
\Psi(w, \theta)=\frac{w}{1-w^{2} / c^{2}} \Omega_{w}-\Omega \\
d\left[\frac{\Omega_{w}}{\rho\left(1-w^{2} / c^{2}\right)}\right]-\frac{\Omega_{\theta}}{p w} d \theta=0 .
\end{gathered}
$$

It is remarkable that Eq. (19) is by no means so easily integrable as Eq. (15) and that Eq. (18) should be compared with only the left-hand side of Eq. (14), the other side being due to the rotationality.

The description of the equations in $\Omega(q, \theta)$ would not be complete without a few words on the Jacobian $J(q, \theta)$. It is necessary to find out where $J$ vanishes because this occurs on the so-called limit lines, i.e., those lines separating the region where the transformation (13) is single-valued from that where it is multi-valued.

To do it easily, a rearrangement of $J$ is convenient. By substituting the quantity $q \Omega_{q}+\Omega_{\theta \theta}$ through its expression which can be isolated from the left-hand side of Eq. (14), $J$ becomes

$$
J(q, \theta)=-\frac{\left(1-q^{2} / a^{2}\right) \Omega_{q q}^{2}+\left(\Omega_{\theta}-q \Omega_{q \theta}\right)^{2} / q^{4}}{1-2 \beta q^{2}\left(1-a^{2} / q^{2}\right) \Omega_{q q} f(\Psi)} .
$$

As in the irrotational case, $J(q, \theta)$ can vanish only if $q>a$, i.e., in the supersonic region of the field. The equation of the limit lines on the plane $(q, \theta)$ is then

$$
\left(1-\frac{q^{2}}{a^{2}}\right) \Omega_{q q}^{2}+\frac{1}{q^{4}}\left(\Omega_{\theta}-q \Omega_{q \theta}\right)^{2}=0 .
$$

Transforming Eq. (14) back in an equation in $\Psi(w, \theta)$ is the second step of this approach. It can be done through Eqs. (15) and (16) and the following substitution formulae

$$
\begin{gathered}
\frac{d q}{d w} \equiv q^{\prime}=\frac{q}{w}\left(1-\frac{w^{2}}{c^{2}}\right) \\
\Omega_{q}=\frac{\Omega_{w}}{q^{\prime}} \\
\Omega_{q q}=\frac{\Omega_{w w}}{q^{\prime 2}}-q^{\prime \prime} \frac{\Omega w}{q^{\prime 3}}
\end{gathered}
$$


(where $q^{\prime \prime} \equiv d^{2} q / d w^{2}$ ) leading to

$$
\frac{w}{q} \Psi_{w}+\int \frac{\psi_{w}}{q} d w+\int \frac{1}{q w}\left(1-\frac{w^{2}}{c^{2}}\right) \Psi_{\theta \theta}=\frac{1}{q} \frac{w^{2} \Psi_{w}^{2}+\left(1-w^{2} / c^{2}\right) \psi_{\theta}^{2}}{w^{2} /\left(2 \beta c^{2}\right)+w \Psi_{w} f(\Psi)} f(\Psi)
$$

which, after multiplication through $q$, derivation with respect to $w$, and substitution of Eq. (11) for $q$, finally becomes

$$
\mathscr{L}(\Psi)=w\left(1-w^{2}\right)^{\beta} \frac{\partial}{\partial w}\left[\frac{1}{w\left(1-w^{2}\right)^{\beta-1}} \frac{\Psi_{w}^{2}+\left(1 / w^{2}-1 / c^{2}\right) \psi_{\theta}^{2}}{1+\Psi_{w} f(\Psi)\left(1-w^{2}\right) / w} f(\Psi)\right]
$$

where $\mathscr{L}(\Psi)$ is the linear operator

$$
\mathscr{L}(\Psi) \equiv w \Psi_{w w}+\left(1+\frac{w^{2}}{c^{2}}\right) \Psi_{w}+\frac{1}{w}\left(1-\frac{w^{2}}{c^{2}}\right) \Psi_{\theta \theta}
$$

It can immediately be noticed that if $f(\Psi)=0 \mathrm{Eq} .(21)$ reduces to $\mathscr{L}(\Psi)=0$, i.e., to the classical Chaplygin equation for irrotational flows.

The Jacobian Eq. (20) becomes

$$
J(w, \theta)=-\frac{1}{w^{2}\left(1-w^{2}\right)^{4 \beta}\left(1-w^{2} / c^{2}\right)} \frac{\Psi_{w}^{2}+\left(1 / w^{2}-1 / c^{2}\right) \Psi_{\theta}^{2}}{1+\Psi_{w} f(\Psi)\left(1-w^{2}\right) / w}
$$

which for $f(\Psi)=0$ reduces to the irrotational form

$$
J_{\text {irr }}(w, \theta)=\frac{\Psi_{w}^{2}+\left(1 / w^{2}-1 / c^{2}\right) \Psi_{\theta}^{2}}{w^{2} \rho^{2}\left(1-w^{2} / c^{2}\right)}
$$

It is interesting to remark that the equation of the limit lines remains the same in both the rotational and irrotational cases

$$
\Psi_{w}^{2}+\frac{1-w^{2} / c^{2}}{w^{2}} \Psi_{\theta}^{2}=0
$$

even if this does not mean that the limit lines are the same, because $\Psi$ will be different in the two cases.

Equation $(21)$ is the main result of this work and it will be used in the following sections to compute some simple cases. It gives the stream function $\Psi(w, \theta)$ when the entropy gradient or the vorticity distribution are assigned through the function $f(\Psi)$.

However, the solution $\Psi(w, \theta)$ is determined on the hodograph plane and must be transferred to the plane $(x, y)$ to give a physical pattern. This transfer, which is only possible in the region external to the limit lines, can be performed by means of Eq. (13) which can be written in terms of $\Psi(w, \theta)$ instead of $\Omega(q, \theta)$ by recalling that

$$
\begin{gathered}
\Omega_{q}=\int \frac{\Psi_{w} d w}{w\left(1-w^{2}\right)^{\beta}} \\
\frac{\Omega_{\theta}}{q}=\int \frac{\Psi_{\theta}}{w^{2}\left(1-w^{2}\right)^{\beta}}\left(1-\frac{w^{2}}{c^{2}}\right) d w .
\end{gathered}
$$

To conclude this section, let us consider the form Eq. (21) assumes when both $w^{2}$ and $w^{2} / c^{2}$ are much smaller than unity, i.e., in the incompressible flow limit. Then

$$
\Delta \Psi=\frac{\partial}{\partial w}\left[\frac{\Psi_{w}^{2}+\Psi_{\theta}^{2} / w^{2}}{w+\Psi_{w} \omega(\Psi)} \omega(\Psi)\right]
$$


where $\Delta$ is the Laplacian operator in the polar coordinates $(w, \theta)$

$$
\Delta \Psi=\Psi_{u w}+\frac{1}{w} \Psi_{w}+\frac{1}{w^{2}} \Psi_{\theta \theta}
$$

and $\omega(\Psi) \equiv f(\Psi)$ is the incompressible vorticity distribution function. The transfer relations from the hodograph to the physical plane are still given by Eq. (13) where now it is

$$
\begin{aligned}
\Omega_{q} & =\int \Psi_{w} \frac{d w}{w} \\
\frac{\Omega_{\theta}}{q} & =\int \Psi_{\theta} \frac{d w}{w^{2}} .
\end{aligned}
$$

2. General solution for a compressible rotational vortex. The nonlinearity of Eq. (21) is a formidable obstacle to its exact solution in the general case. However, there is at least one situation in which this difficulty can be overcome, i.e., the circular vortex flow. Here a vortical motion is meant in which the vorticity, instead of being concentrated at the centre as an unbounded peak, is spread overall in the flow field, i.e., a rotational vortex. Even if the author can hardly believe that this mathematical flow pattern is new, he is nevertheless unable to find it in the literature on the compressible flow of an ideal fluid. This reason and also the fact that this flow looks particularly suitable for a treatment which makes use of the above derived equations suggest to describe it extensively.

Let us look for solutions of Eq. (21) having the form $\Psi=\Psi(w)$, i.e., not depending on $\theta$. Then only $\Omega_{q}$, as given by the first of Eqs. (22), enters Eq. (13). Hence

$$
r=\left(x^{2}+y^{2}\right)^{1 / 2}= \pm \int \frac{\Psi_{w} d w}{w\left(1-w^{2}\right)^{\beta}} .
$$

This relation can be differentiated to give

$$
\frac{d \Psi}{d w}= \pm w\left(1-w^{2}\right)^{\beta} \frac{d r}{d w}
$$

which enables one to obtain $\Psi(w)$ if the function $r(w)$ is assigned. The ambiguity of the algebraic sign can be eliminated by observing that only the solution with the minus sign identically satisfies Eq. (21) and therefore is the one to be retained.

As a consequence it can be stated that every speed distribution $w(r)$ represents a compressible vortical flow whose stream function is (in these and in the following relations all $K$ 's are constants)

$$
\begin{gathered}
\Psi(w)=-\int \frac{w\left(1-w^{2}\right)^{\beta}}{d w / d r} d w+K_{1} \\
\Psi(r)=-\int w\left(1-w^{2}\right)^{\beta} d r+K_{2}
\end{gathered}
$$

on the hodograph and on the physical planes, respectively. The vorticity distribution is given by the kinematical formula

$$
\omega(r)=\frac{w}{r}+\frac{d w}{d r}
$$

which is of course the same in both compressible and incompressible cases. 
Equations (23) are wholly general and hold for every $w(r)$ so far as $w \leq 1$, the unit value meaning limit (cavitation) speed. This limitation is not new: it appeared already in the irrotational vortex described by Taylor [3] and Ringleb [4]. In that case $\omega(r)=0$ and therefore

$$
w=\frac{\Gamma}{2 \pi r}
$$

where $\Gamma$ is the (constant) circulation. Eqs. (23) then give

$$
\begin{gathered}
\Psi(w)=\frac{\Gamma}{2 \pi} \int \rho \frac{d w}{w}+K_{1}^{\prime} \\
\Psi(r)=-\frac{\Gamma}{2 \pi} \int\left(1-\frac{\Gamma^{2}}{4 \pi^{2} r^{2}}\right)^{\beta} \frac{d r}{r}+K_{2}^{\prime} .
\end{gathered}
$$

For $r<\Gamma /(2 \pi)$ the second of these two relations loses its meaning and a central core has to be accepted in which no flow is possible.

A similar, though less drastic, situation occurs in the incompressible case where $w$ becomes unbounded as $r \rightarrow 0$ and a central core must also be excluded from the irrotational flow field. A classical remedy to this was proposed by Rankine and consists in the substitution of the flow region inside an assigned radius $r_{0}$ through a rotational vortex characterized by a rigid speed distribution

$$
w=\frac{\omega}{2} r
$$

with $\omega$ constant. Of course a general speed distribution (associated to the statement of a constant total enthalpy) could be justified only by admitting a suitable nonconservative force distribution acting on the fluid. As in the incompressible case, however, selecting the rigid speed distribution allows one to identify this nonconservative force system in the viscous stresses which would be acting if the fluid were viscous, which makes this speed distribution rather realistic. Furthermore, introduction of Eq. (25) into Eqs. (23) leads to expressions which can be exactly integrated

$$
\begin{gathered}
\Psi(w)=\frac{\left(1-w^{2}\right)^{\beta+1}}{(\beta+1) \omega}+K_{1}^{\prime \prime} \\
\Psi(r)=\frac{1}{(\beta+1) \omega}\left(1-\frac{\omega^{2}}{4} r^{2}\right)^{\beta+1}+K_{2}^{\prime \prime} .
\end{gathered}
$$

Now the limit speed is never attained so far as $r \leq 2 / \omega$. It is therefore possible to choose a radius $r_{0}$ intermediate between $\Gamma /(2 \pi)$ and $2 / \omega$ in order to generate a compressible Rankine vortex. By remembering that $\Gamma$ and $\omega$ are related through $\Gamma=\omega \pi r_{0}^{2}$, it then turns out that only the condition $r_{0} \leq 2 / \omega$ is necessary because it also automatically implies that $r_{0} \geq \Gamma /(2 \pi)$.

In Fig. $1 \Psi(r)$ and $w(r)$ are represented for the case $\omega=1, r_{0}=1, \beta=\frac{5}{2}$. This value of $\beta$ (valid for air and all diatomic gases) allows one to analitically perform the integral in the second of Eqs. (24) giving

$$
\Psi(r)=\frac{\omega}{2} r_{0}^{2}\left[\frac{R^{5}}{5 r^{5}}+\frac{R^{3}}{3 r^{3}}+\frac{R}{r}-\ln (r+R)\right]+K_{2}^{\prime}
$$


with $R=\left(r^{2}-\omega r_{0}^{2} / 2\right)^{1 / 2}$. In Fig. 1 also the curve for the function $\Psi_{\text {inc }}(r)$ of the incompressible case with the same $\omega, r_{0}$ is reported for comparison, where $\Psi_{\text {inc }}=$ $-r^{2} / 4$ if $r \leq r_{0}$ and $\Psi_{\text {inc }}=-\left(\frac{1}{2}+\ln r\right) / 2$ if $r \geq r_{0}$.

A few words on the opposite case in which $\Psi=\Psi(\theta)$, i.e., the source/sink flow, may conclude this section. If $\Psi$ is only a function of $\theta$ the right-hand side of Eq. (21) vanishes and the equation collapses to the irrotational case. As a consequence every $\Psi(\theta)$ representing an irrotational source/sink flow represents also an infinity of analogous rotational flows provided that the stream function is interpreted in the generalized sense of Eq. (4).

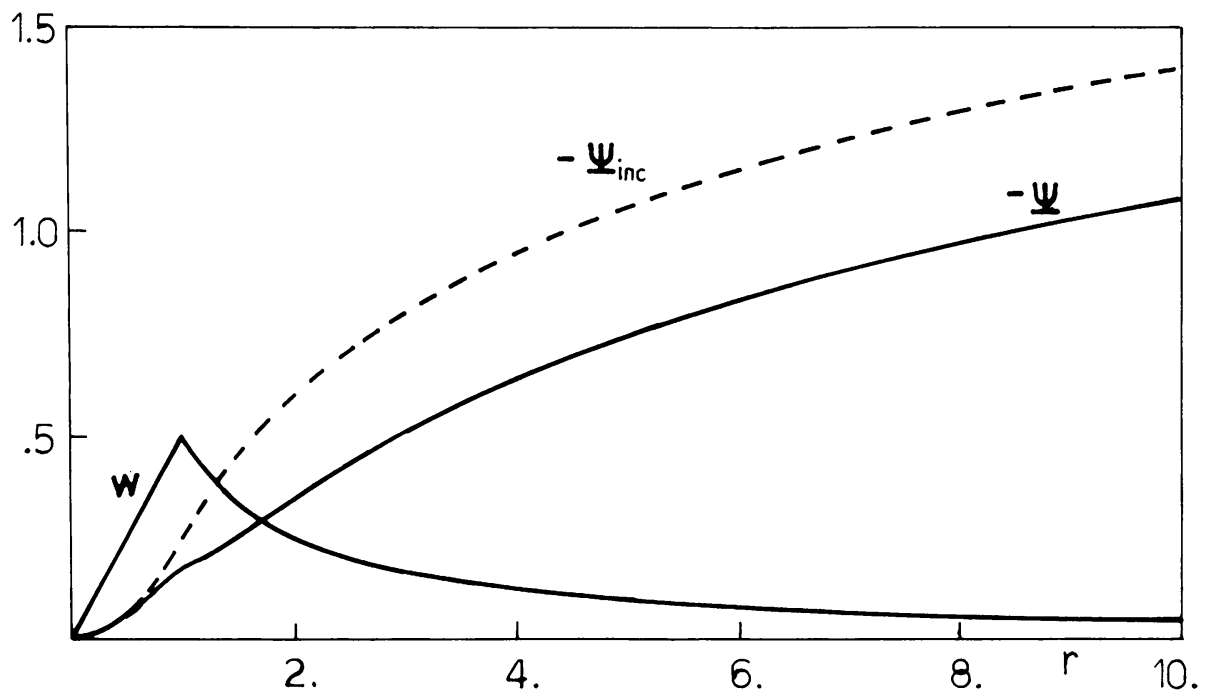

Fig. 1. Velocity and stream function versus radius $r$ in a compressible Rankine vortex for $\omega=1, r_{0}=1, \beta=\frac{5}{2}$. Constants $K_{2}^{\prime}$ and $K_{2}^{\prime \prime}$ are given values -0.344084 and $-\frac{2}{7}$, respectively, in order to make both internal and external $\Psi$ 's match each other at $r=r_{0}$. Dashed line: incompressible case.

3. Perturbative formulation for a weakly rotational flow. In some practical cases the entropy gradient is small. This is the case for example of the region behind a shock wave if both strength and curvature of the shock are sufficiently low. Then the amount of vorticity injected downstream is also small, and the same is true for the values of the function $f(\Psi)$ given by Eq. (7). As a consequence the whole right-hand side of Eq. (21) is small and the effect of the entropy gradient is tantamount to a perturbation of the irrotational flow pattern described by the left-hand side of the same Eq. (21) when isolated and put equal to zero. The latter being a linear form in $\Psi$, this assumption leads to the linearization of the problem.

Let us assume that $\varepsilon$ is a representative value for the magnitude of $f(\Psi)$ and that $\varepsilon \ll 1$. By writing

$$
f(\Psi)=\varepsilon \bar{f}(\Psi)
$$


the function $\bar{f}(\Psi)$ has the order of unity. Let us furthermore look for the solution $\Psi$ in the form

$$
\Psi=\Psi_{0}+\varepsilon \Psi_{1}+\varepsilon^{2} \Psi_{2}+\cdots
$$

where $\Psi_{0}$ is the irrotational (unperturbed) solution and all $\Psi_{i}$ 's have the order of unity. Expanding $f(\Psi)$ now in a Taylor series in a neighbourhood of $\Psi_{0}$ gives

$$
f(\Psi)=f\left(\Psi_{0}\right)+f^{\prime}\left(\Psi_{0}\right)\left(\Psi-\Psi_{0}\right)+\frac{1}{2} f^{\prime \prime}\left(\Psi_{0}\right)\left(\Psi-\Psi_{0}\right)^{2}+\cdots
$$

or, after collecting all terms with the same order in $\varepsilon$ and using Eq. (26)

$$
f(\Psi)=\varepsilon \bar{f}_{0}+\varepsilon^{2} \bar{f}_{0}^{\prime} \Psi_{1}+\varepsilon^{3}\left(\bar{f}_{0}^{\prime} \Psi_{2}+\frac{1}{2} \bar{f}_{0}^{\prime \prime} \Psi_{1}^{2}\right)+\cdots
$$

where $\bar{f}_{0}$ stands for $\bar{f}\left(\Psi_{0}\right), \bar{f}_{0}^{\prime}$ for $\bar{f}^{\prime}\left(\Psi_{0}\right)$, etc. Owing to the linearity of the operator $\mathscr{L}(\Psi)$ Eq. (33), it is possible to write

$$
\mathscr{L}(\Psi)=\varepsilon \mathscr{L}\left(\Psi_{1}\right)+\varepsilon^{2} \mathscr{L}\left(\Psi_{2}\right)+\varepsilon^{3} \mathscr{L}\left(\Psi_{3}\right)+\cdots
$$

where it has been already taken into account that $\mathscr{L}\left(\Psi_{0}\right)=0$. By now introducing Eqs. (27) and (28) into Eq. (21) and equating all terms having the same order in $\varepsilon$, the complete nonlinear problem in $\Psi$ can be split in a sequence of linear problems in $\Psi_{1}, \Psi_{2}, \ldots$. In doing this, attention should be paid to the fact that the expansion of the right-hand side of Eq. (21) in powers of $\varepsilon$ converges only if

$$
\left|\varepsilon \Psi_{w} \bar{f}(\Psi)\left(1-w^{2}\right) / w\right|<1
$$

which states a lower speed limit (depending on $\theta$ ) to the validity of this procedure. Once this condition is satisfied, the first two problems can be so formulated:

- perturbation of order $\varepsilon$

$$
\mathscr{L}\left(\Psi_{1}\right)=w\left(1-w^{2}\right)^{\beta} \frac{\partial}{\partial w}\left[\frac{1}{w\left(1-w^{2}\right)^{\beta-1}}\left(\Psi_{0 w}^{2}+\frac{1-w^{2} / c^{2}}{w^{2}} \Psi_{0 \theta}^{2}\right) \bar{f}_{0}\right]
$$

- perturbation of order $\varepsilon^{2}$

$$
\begin{aligned}
\mathscr{L}\left(\Psi_{2}\right)=w\left(1-w^{2}\right)^{\beta} \frac{\partial}{\partial w}\{ & \frac{1}{w\left(1-w^{2}\right)^{\beta-1}} \\
\times & {\left[\left(\Psi_{0 w}^{2}+\frac{1-w^{2} / c^{2}}{w^{2}} \Psi_{0 \theta}^{2}\right)\left(\bar{f}_{0}^{\prime}-\frac{1-w^{2}}{w} \bar{f}_{0}^{2} \Psi_{0 w}\right)\right.} \\
& \left.\left.+2 \bar{f}_{0}\left(\Psi_{0 u} \Psi_{1 w}+\frac{1-w^{2} / c^{2}}{w^{2}} \Psi_{0 \theta} \Psi_{1 \theta}\right)\right]\right\}
\end{aligned}
$$

where $\Psi_{1}$ comes from the solution of the first-order problem.

The right-hand sides of these equations are not necessarily different from zero on the whole flow field. For instance, in the case of the transonic flow on a wing profile with an attached shock wave having finite extension, only the region extending from the shock to the downstream infinity is rotational and contributes to $\Psi_{1}, \Psi_{2}, \ldots$.

4. A rotationally perturbed Ringleb flow. From the practical standpoint the most interesting cases for the application of the method of Sec. 3 are the transonic flows with shocks around wing airfoils quoted above. However, their computation requires 
a lot of numerics only to obtain the irrotational solution $\Psi_{0}$ to start with (see, e.g., [5]). The latter must then be transferred on the hodograph plane and there perturbed by means of Eq. (30) and the subsequent ones. This step would also involve a remarkable numerical work.

It is likely that this numerical effort would not exceed that commonly requested by all current transonic flow computations, which perhaps makes the present alternative worth checking. In the framework of this investigation, however, a more fundamental point of view has been assumed, by selecting a case suitable for a fully analytical treatment. This can only be achieved by means of a simple irrotational flow and a simple perturbation function $f(\Psi)$; even if the result will not be relevant for direct practical use, its validity field will be well assessed. It is therefore possible to suggest its use as a test case to check Euler computations.

The irrotational flow here selected as zero-order solution is the Ringleb flow [4]

$$
\Psi_{0}=\frac{\sin \theta}{w}
$$

which represents the $180^{\circ}$ deflection of a semi-infinite stream around the tip of a slender symmetrical body extending back to infinity. A peculiar feature of this flow is the occurrence of a supersonic region on the tip, which makes the Ringleb flow a transonic one.

The selected perturbation is related to an entropy distribution depending linearly on $\Psi$

$$
\frac{S}{R}=\sigma \Psi+S_{0}
$$

where $\sigma$ and $S_{0}$ are constants. This implies

$$
f\left(\Psi_{0}\right)=\frac{\sigma}{2(\beta+1)}=\varepsilon
$$

because of Eq. (7). The function $f\left(\Psi_{0}\right)$ is therefore a constant and $\varepsilon$ is its value. Then Eq. (26) immediately gives $\bar{f}_{0}=f\left(\Psi_{0}\right) / \varepsilon=1$ and the first-order perturbative equation (30) becomes, after substitution of Eq. (3) for $c^{2}$ and of Eq. (31) for $\Psi_{0}$,

$$
\mathscr{L}\left(\Psi_{1}\right)=\phi_{0}(w)+\phi_{2}(w) \cos 2 \theta
$$

where

$$
\begin{aligned}
& \phi_{0}(w)=-\frac{(1+\beta)(2 \beta+3) w^{4}-(5 \beta+8) w^{2}+5}{w^{5}\left(1-w^{2}\right)} \\
& \phi_{2}(w)=-\beta \frac{(2 \beta+3) w^{2}-3}{w^{2}\left(1-w^{2}\right)} .
\end{aligned}
$$

The particular form of Eq. (32) suggests to look for solutions like

$$
\Psi_{1}(w, \theta)=\boldsymbol{\Phi}_{0}(w)+\boldsymbol{\Phi}_{2}(w) \cos 2 \theta
$$

where $\Phi_{0}$ and $\Phi_{2}$ must satisfy to the following ordinary differential equations

$$
\begin{gathered}
w \Phi_{0}^{\prime \prime}+\left(1+\frac{w^{2}}{c^{2}}\right) \Phi_{0}^{\prime}=\phi_{0}(w) \\
w \Phi_{2}^{\prime \prime}+\left(1+\frac{w^{2}}{c^{2}}\right) \Phi_{2}^{\prime}-\frac{4}{w}\left(1-\frac{w^{2}}{c^{2}}\right) \Phi_{2}=\phi_{2}(w) .
\end{gathered}
$$


No particular assumption on the value of $\beta$ has been made till now. As the solutions of Eqs. (34) involve certain integrals which can be exactly performed only if $\beta$ is a half-integer, all computations in the following assume that $\beta=\frac{5}{2}$ : this means considering a general diatomic gas, including the important case of the air. The solution to the first of Eqs. (34) is then

$$
\begin{aligned}
\Phi_{0}(\dot{w})= & \frac{7}{8} w^{4}-\frac{7}{6} w^{2}-\frac{119}{32}+\frac{37}{16 w^{2}}-\frac{5}{16 w^{4}} \\
& -\frac{35}{64}\left(\ln \frac{1+\left(1-w^{2}\right)^{1 / 2}}{1-\left(1-w^{2}\right)^{1 / 2}}\right)^{2}+\left(C_{1}-\frac{35}{16} \ln \frac{1+\left(1-w^{2}\right)^{1 / 2}}{1-\left(1-w^{2}\right)^{1 / 2}}\right) \\
& \cdot\left[\frac{\left(1-w^{2}\right)^{1 / 2}}{5}\left(w^{4}-\frac{11}{3} w^{2}+\frac{23}{3}\right)-\frac{1}{2} \ln \frac{1+\left(1-w^{2}\right)^{1 / 2}}{1-\left(1-w^{2}\right)^{1 / 2}}\right]+C_{2}
\end{aligned}
$$

while $\Phi_{2}(w)$ is obtainable if the fundamental solutions to the second of Eqs. (34) are known [6]

$$
\begin{aligned}
\Phi_{2}(w)= & \mathscr{F}_{1}(w) \int_{w_{1}}^{w} \frac{\phi_{2}\left(w^{\prime}\right) \mathscr{F}_{2}\left(w^{\prime}\right) d w^{\prime}}{w^{\prime} D\left(w^{\prime}\right)}-\mathscr{F}_{2}(w) \int_{w_{1}}^{w} \frac{\phi_{2}\left(w^{\prime}\right) \mathscr{F}_{1}\left(w^{\prime}\right) d w^{\prime}}{w^{\prime} D\left(w^{\prime}\right)} \\
& +C_{3} \mathscr{F}_{1}(w)+C_{4} \mathscr{F}_{2}(w) .
\end{aligned}
$$

Quantities $C_{i}$ and $w_{1}$ are constants to be assigned; moreover

$$
\begin{aligned}
\mathscr{F}_{1}(w) & =w^{2} F\left(\frac{5}{2},-3,3 ; w^{2}\right) \equiv w^{2} F_{2}\left(w^{2}\right) \\
\mathscr{F}_{2}(w) & =\frac{1}{w^{2}} F\left(\frac{1}{2},-5,1 ; w^{2}\right) \equiv \frac{1}{w^{2}} F_{-2}\left(w^{2}\right) \\
D(w) & =\mathscr{F}_{1}(w) \frac{d \mathscr{F}_{2}}{d w}-\mathscr{F}_{2}(w) \frac{d \mathscr{F}_{1}}{d w} \\
& =-\frac{4}{w} F_{2} F_{-2}+2 w\left(F_{2} F_{-2}^{\prime}-F_{2}^{\prime} F_{-2}\right)
\end{aligned}
$$

$F_{n}\left(w^{2}\right)$ being the hypergeometric function of order $n$ and $F_{n}^{\prime} \equiv d F_{n} / d\left(w^{2}\right)$.

There are some degrees of freedom in the choice of the $C_{i}$ and $w_{1}$ but a request should be satisfied in every case. It appears from both exact equations (2) and (21) that vorticity has no influence on the irrotational flow where conditions are sonic. This property should be preserved by imposing that the perturbation vanishes along the sonic line, i.e., where $w=w^{*}$ with the sonic speed $w^{*}$ given by $w^{*}=(2 \beta+1)^{-1 / 2}$ which reduces to $1 / \sqrt{6}$ for air and other diatomic gases.

This is achieved by assuming

$$
\begin{gathered}
w=w^{*} \\
C_{1}=\frac{35}{16} \ln (11+2 \sqrt{30}) \\
C_{2}=\frac{91}{72}+\frac{35}{64}[\ln (11+2 \sqrt{30})]^{2} \\
C_{3}=C_{4}=0 .
\end{gathered}
$$

The selected values of $C_{1}$ and $C_{2}$ cause $\Phi_{0}\left(w^{*}\right)$ to be zero (of course many other choices would be possible). The perturbed stream function on the hodograph plane 
then results from the sum of $\Psi_{0}$, Eq. (31), and of $\varepsilon \Psi_{1}$ with $\Psi_{1}$ given by Eq. (33). The streamline equation can then be made explicit in the form $\theta=\theta(w)$

$$
\sin \theta=\frac{1}{4 \varepsilon \Phi_{2} w}\left\{1-\left[1-8 \varepsilon \Phi_{2} w^{2}\left(\Psi-\varepsilon \Phi_{0}-\varepsilon \Phi_{2}\right)\right]^{1 / 2}\right\} .
$$

The transfer to the physical plane can now be performed by means of Eqs. (13). It results in

$$
\begin{aligned}
& x=x_{R}-\varepsilon\left(S_{0} \sin \theta+S_{1} \sin \theta \cos 2 \theta-S_{2} \sin 2 \theta \cos \theta\right) \\
& y=y_{R}+\varepsilon\left(S_{0} \cos \theta+S_{1} \cos \theta \cos 2 \theta+S_{2} \sin 2 \theta \sin \theta\right)
\end{aligned}
$$

where $x_{R}$ and $y_{R}$ are the coordinates in the unperturbed Ringleb flow

$$
\begin{aligned}
& x_{R}=-\frac{5}{2}\left(\frac{1}{2} \ln \frac{1+u}{1-u}-\frac{1}{u}-\frac{1}{3 u^{3}}-\frac{1}{5 u^{5}}\right)+\frac{\cos 2 \theta}{2 \rho w^{2}} \\
& y_{R}=\frac{\sin 2 \theta}{2 \rho w^{2}}
\end{aligned}
$$

and

$$
\begin{gathered}
u=\left(1-w^{2}\right)^{1 / 2} \\
S_{0}=\frac{5}{12 w^{5} u^{3}}-\frac{5 w^{3}}{4 u^{3}}-\frac{22 w}{3 u}-\frac{173 u}{24 w}-\frac{23 u^{3}}{24 w^{3}} \\
-\frac{2 u^{5}}{3 w^{5}}+\frac{36}{16 w} \ln \frac{1+u}{1-u}-\frac{C_{1}}{w}+C_{5} \\
S_{1}=\frac{\Phi_{2}(w)}{w u^{5}}+\frac{S_{2}}{2} \\
S_{2}=2 \int_{w_{2}}^{w} \frac{1-6 w^{2}}{w^{2} u^{7}} \Phi_{2}(w) d w .
\end{gathered}
$$

Two new constants $w_{2}$ and $C_{5}$ appear here. For their determination the condition of vanishing perturbation on the sonic line can be applied again, which leads one to assume

$$
\begin{gathered}
w_{2}=w^{*} \\
C_{5}=\frac{515}{12 \sqrt{5}}
\end{gathered}
$$

where the value for $C_{5}$ is the one for which $S_{0}\left(w^{*}\right)=0$.

The obtained flow has the same sonic line as the unperturbed one but is different elsewhere. Due to the singular terms in $\Phi_{0}$, Eq. (35), the perturbation tends to grow unboundedly when low speed regions are approached: this confines the validity of the present expansion to the transonic region (which is fortunately the interesting one). From the physical standpoint this means that every perturbation, no matter how small, is dominant where the flow itself is vanishing. From the mathematical standpoint, this fact had to be expected because low values of $w$ invalidate the convergence condition Eq. (29) which becomes in this case

$$
|\sin \theta|<\left|-\frac{1}{\varepsilon} \frac{w^{3}}{1-w^{2}}\right| \text {. }
$$


Computations have been performed for $\varepsilon=-0.01$, corresponding to an entropy gradient $d(S / R) / d \Psi=-0.07$. Double precision arithmetic has been used, where the hypergeometric functions appearing in Eq. (37) have been evaluated through a procedure due to Chiocchia and Gabutti [7]. The resulting flow pattern is represented in Fig. 2 limited to the region where condition Eq. (37) is satisfied. It turns out that a value $w_{\min }$ can be found making the right-hand side of Eq. (37) equal to unity: it states therefore an absolute lower limit to $w$ because every $w>w_{\min }$ satisfies Eq. (37) independently from the value of $\theta$. Being in this case $w_{\min }=.212161$, the flow represented in Fig. 2 lies entirely inside the converging region. In terms of Mach number this limit becomes $M_{\min }=.485458$ (of course both $w_{\min }$ and $M_{\min }$ could be lowered by selecting smaller values of $\varepsilon$ ).

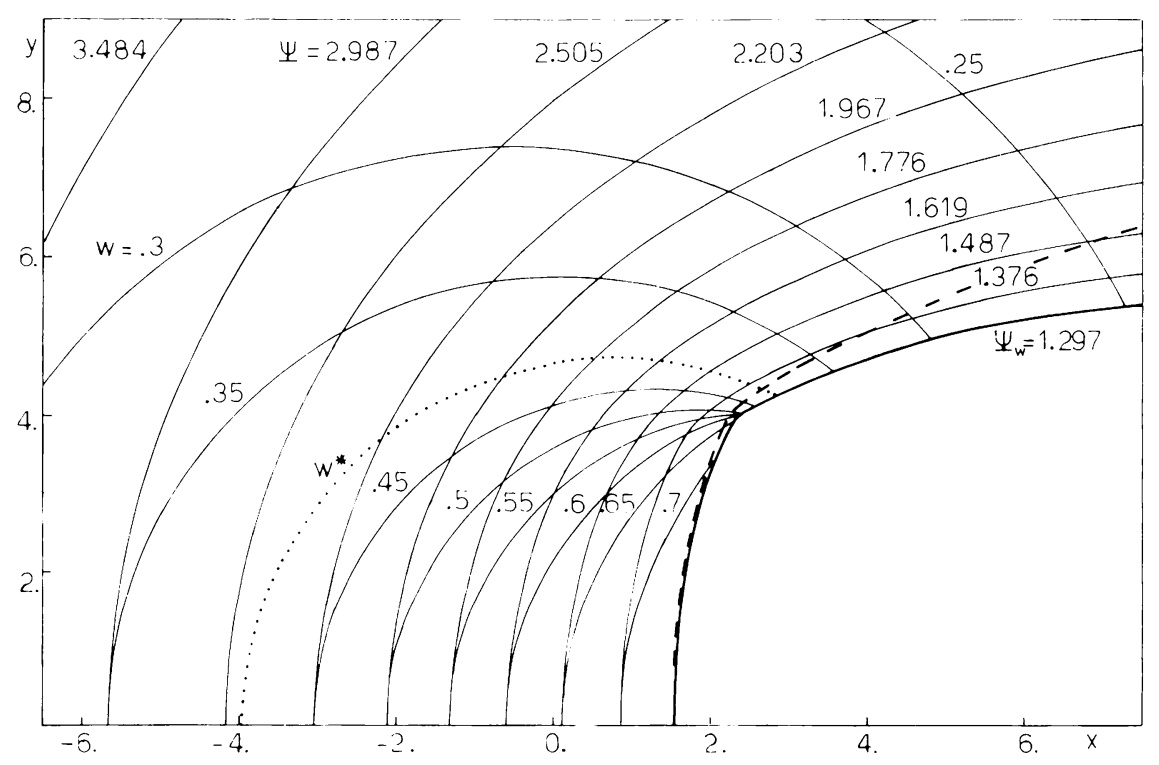

FIG. 2. Streamlines and isotach lines in the transonic region of the perturbed Ringleb flow for $\varepsilon=-0.01, \beta=\frac{5}{2}$ (the sonic line is dotted). Dashed line: wall contour of the original (irrotational) Ringleb flow.

The most visible differences with respect to the irrotational Ringleb flow are:

- whereas the sonic line is the same as the irrotational one and therefore conserves its circular form, all other isotach lines are no more circular and appear more spaced, both in the subsonic and in the supersonic region;

- as in the irrotational case, limit lines appear in the inner region of the field which can be excluded by selecting a suitable streamline as a rigid wall: the rotational flow around the tip of a rounded body is so described;

- the streamline slope in the subsonic region decreases faster than in the irrotational case and the resulting body shape is more slender. This tendency can be considered realistic only so far as the above discussed lower speed limit is not attained; 
- the highest speed is reached on the tip and amounts to 0.741 corresponding to a Mach number of 2.467, while in the irrotational case these values are 0.745 and 2.5 respectively, i.e., fully comparable;

- the maximum speed is read on the innermost streamline not crossing the limit lines ( $\Psi \equiv \Psi_{w}=1.297$ in this case). This streamline presents a point of discontinuous curvature where isotach lines converge giving infinite acceleration (visible in Fig. 2). A more regular wall contour could of course be selected by choosing any streamline for which $\Psi>\Psi_{w}$;

- as in the irrotational case, streamlines converge in the subsonic region and diverge in the supersonic one when moving towards the symmetry axis. This fact can be given a simple explanation in the irrotational case because of mass flow conservation. In the rotational case, however, the same simple argument cannot be invoked, because Eq. (5) does not hold any more.

Conclusions. In this work a procedure has been illustrated to compute rotational adiabatic compressible flows of ideal nonviscous fluids. The procedure is indirect, in that it derives the shape of the rigid boundaries, rather than assuming them. However, a perturbative method has also been presented, allowing for the modification of a preexisting irrotational flow field and an application has been shown in which the Ringleb flow has been assumed as the unperturbed one.

These results, valid in a sufficiently wide transonic region, are not purely of academic interest. A rotational Ringleb flow was in fact already computed by L. Zannetti [8] by means of a finite difference numerical scheme. However, the computations assumed a constant stagnation pressure and a variable stagnation enthalpy: it could in fact be demonstrated that this assumption made the flow rotational by leaving boundaries and streamlines unchanged. By superposing a stagnation enthalpy gradient normal to the streamlines to the original Ringleb flow, it was therefore possible to compute pressures, densities and velocities for a new rotational flow having the same boundaries and the same stagnation pressure as the former one. The present work's approach is different in that the stagnation enthalpy is everywhere constant (its value being that of the points of the unperturbed sonic line); the stagnation pressure is, on the contrary, free to adapt itself to the new conditions. These features are characteristic of the region behind a shock wave in a transonic flow and therefore should make the presented results interesting also for code testing.

\section{REFERENCES}

[1] L. Crocco, Una nuova funzione di corrente per lo studio del moto rotazionale dei gas, Rendiconti R. Acc. Naz. Lincei XXIII (2), (1936)

[2] C. Ferrari and F. Tricomi, Transonic Aerodynamics, Academic Press, New York, and London, 1968

[3] G. I. Taylor, Recent work on the flow of compressible fluids, J. London Math. Soc. 5, 224 (1930)

[4] F. Ringleb, Exakte Lösungen der Differentialgleichungen einer adiabatischen Gasströmung, Z. Angew. Math. Mech. 20 4, 185-198 (1940) 
[5] A. Jameson, Iterative solution of transonic flows over airfoils and wings, including flows at Mach 1, Comm. Pure Appl. Math. XXVII, 283-209 (1974)

[6] P. Germain and R. Bader, Solutions élementaires de certaines équations aux derivées partielles du type mixte, Bull. Soc. Math. de France 81, 145-174 (1953)

[7] G. Chiocchia and B. Gabutti, A new transformation for computing hypergeometric series and the exact evaluation of the transonic adiabatic flow over a smooth bump, Comput. \& Fluids 17 1, 13-23 (1989)

[8] L. Zannetti, $A$ natural formulation for the solution of two-dimensional or axisymmetric inverse problems, Int. J. Num. Math. Eng. 22, 451-453 (1986) 